\title{
Eiszeitliche Mittelmoränen in der Zentral- und Ostschweiz
}

\author{
René HanTKE*)
}

Hantke, R. (2003): Eiszeitliche Mittelmoränen in der Zentral- und Ostschweiz. - Eiszeitalter und Gegenwart, 52: 13-24; Hannover.

Keywords: ice age, Linth glacier, medial moraines, Muota glacier, east Switzerland, Reuss glacier, Sihl glacier, Würm glacial, central Switzerland

Kurzfassung: Bei der Kartierung für den Geologischen Atlas der Schweiz 1: 25000 in der Zentralschweiz und in den westlichen Glarner Alpen wurden viele Quartärstrukturen als Ansätze von Mittelmoränen erkannt. Mittelmoränen, gebildet aus zwei Seitenmoränen am Zusammenfluss zweier Gletscher, wurden in der Eiszeitliteratur bisher viel zu wenig beachtet. $\mathrm{Als}$ Obermoränen lassen sie sich meist bis ans Zungenende verfolgen. Im proximalen Bereich liefern Mittelmoränen Hinweise auf die Eishöhen. Im distalen Bereich sind viele bisher als Drumlin kartierte Bildungen als Mittelmoränen zu deuten. Übereinander gelegene Abfolgen zeigen, dass Mittelmoränen sich oft über mehrere Kaltzeiten an den selben Stellen eingestellt haben. Ebenso lassen sich in ihrer Lage schwer verständliche, isolierte Schotter-Vorkommen auf Hochflächen des Mittellandes als von epiglaziären Schmelzwässern verschwemmtes Schuttgut auf Grund gelaufener Mittelmoränen erklären. Bisher wurden solche meist in der Schweiz als Überreste von alten Schotterfluren, als Deckenschotter-Vorkommen, gedeutet. In diese zusammenhängende Flur hätten sich später die Täler sukzessive eingetieft, was jedoch nie belegt worden ist.

\section{[Medial moraines in Central and Eastern Switzerland]}

Abstract: During cartographic field work for the Swiss Geological Atlas 1: 25000 in Central Switzerland and in the western Glaronese Alps, many incipient moraine structures have been recognized as medial moraines. Medial moraines are poorly covered in the litterature, although they take up the combined debris of two lateral moraines at the confluence of two glaciers. In most cases they can be followed up to the end of the glacier as superficial moraines. Medial moraines furnish information

*) Anschrift des Verfassers: Prof. Dr. René HantKe, Glärnischstr. 3, CH-8712 Stäfa ZH. regarding the altitude of the ice ccver. In the distal area many forms which have been regarded as drumlins, have to be interpreted as medial moraines. Superposed sequences show that medial moraines have been formed at the same place during several sold periods. Isolated occurrences of gravel on high plate zus of the Midland in positions difficult to understand are also most easily to interpret as debris of agrounded midial moraine, retransported by meltwater. Hitherto they were interpreted in Switzerland as rests of former hi her gravel plains, as "Deckenschotter." Into these coh rent gravel plains at high altitude the valleys of the Midland have been cut according to a hypothesis never proven.

\section{Einleitun $y$}

Seit 20 Jahren hat sich WAGNER $(1986,1997,2001)$ mit dem bisher recht stiefmüt terlich behandelten Problem eiszeitlicher Mittelmoränen auseinander gesetzt. Ein alpines Gletschersy: tem, das aus n TeilGletschern besteht, erhält an den Vereinigungsstellen insgesamt n-1 Mittel noränen. Die aus zahlreichen kleinen und grosien Teil-Gletschern bestehenden pleistozänen alpinen Gletscher haben daher deren viele besessen. In den Schweizer Alpen ragten in allen Kaltzeiten Felsgipfel bis über 1000 m über die Firnflächen empor, so dass auf diese selbst in den Höchstständen Gesteins schutt niederbrach.

An Konfluenzen von Teil-Glet chern übernahmen Mittelmoränen den primàes Randschutt und führten diesen auf einem eisigen Förderband bis ans Ende oder an den Rand der Gletscherzunge. WAGNER beschäftigt sich mit terminalen und lateralen Endaufschüttungen der Mittelmoränen im AlpenVorland und in den Alpentälern. In der vorliegenden Arbeit werden inneralpine Vereinigungsstellen von Teil-Gletschern betrachtet: die Ansatzstellen, der Ursprung der Mittelmoränen. Es wird gezeigt, dass ein Teil des primären Randschuttes von TeilGletschern nicht auf die entstehende Mittelmoräne gelangte, sondern an den Ansatzstellen als charakteristische Ablagerungen liegen blieb. Diese Stellen geben Hinweise über Eishöhen von Höchstständen 
und Wiedervorstössen. Noch in HantKe (197883) wurde den Mittelmoränen viel zu wenig Beachtung geschenkt. Bei der Druckbereinigung der Blätter Rigi, Einsiedeln, Muotatal, Linthal, Klöntal und Ibergeregg des Geologischen Atlas der Schweiz 1:25000, bei letzten Begehungen und beim Verfassen der Erläuterungstexte mehrten sich im Kontakt mit WaGner die Erkenntnisse um diese wichtigen landschaftsgestaltenden Elemente, sowohl bei ihrem Ansatz wie an ihrem Ende (HANTKE et al. 2000a-f). Auch in Kaltzeiten wurde der Großteil des Schuttgutes wie bei heutigen alpinen Gletschern auf dem Eis transportiert und nicht, wie bisher angenommen, sub- und endoglaziär. Die Mittelmoränen-Schuttbänder flachten gegen die Zungenenden $a b$, rückten näher zusammen oder vereinigten sich und gelangten als Obermoräne und Schotter in typischen Strukturen zur Ablagerung; WAGNER (1997) hat sie als Mittelmoränen-Quappen bezeichnet und eine Typologie aufgestellt. Im Ablationsgebiet kommt dem Transport durch epiglaziäre Schmelzwässer zunehmende Bedeutung zu: Neben reinen Moränen treten oft über kurze Distanzen verfrachtete Schotter, verschwemmte Mittelmoränen, auf (HANTKE \& WAGNER 2002).

\section{Zwei altbekannte Mittelmoränen-Ansätze}

\subsection{NW-Nase des Bürgenstock und ihre subaquatische Fortsetzung in den Vierwaldstätter See}

Altbekannt ist die mit Erratikern garnierte Mittelmoräne auf der NW-Nase des Bürgenstock (LK 1170 Alpnach, Atlasblatt Luzern) und ihre subaquatische Fortsetzung im Vierwaldstätter See. Sie bildete sich zwischen dem durch die Wannen des Vierwaldstätter Sees abgeflossenen Reuss-Gletscher und dem über den Brünig geflossenen Arm des Aare-Gletschers. Dieser reichte in der letzten Eiszeit auf dem $1000 \mathrm{~m}$ hohen Pass bis auf $1700 \mathrm{~m}$. Bei Stans vereinigte er sich mit dem linken Arm des Engelberger Gletschers.

Von Kehrsiten lässt sich der bis $60 \mathrm{~m}$ aufragende, schon von BuXTORF (1910, BuXTORF et al. 1916) und Kopp (Kopp et al. 1955, 1962) als Mittelmoräne gedeutete Wall subaquatisch $1,5 \mathrm{~km}$ in den Vierwaldstätter See gegen Luzern verfolgen (Tab. 1, HANTKE 2003b).

\subsection{Chammhalden am Säntis}

An der NW-Flanke des Säntis, in den Chammhalden (HantKe 1978, Fig. 29, S. 86; 1980, Fig. 43, S. 85; in Funk et al., 2000; Abb. 2, Tab. 1), hat sich durch Frostsprengung und Steinschlag eine klassische Mittelmoräne gebildet. Ihre Schüttung erfolgte über alle Kaltzeiten seit der Platznahme der helvetischen Säntis-Schuppen vor 5 Millionen Jahren zwischen einem gegen NW abfliessenden Urnäsch- und einem gegen NE sich wendenden WissbachGletscher, einem Zufluss des Sitter-Gletschers. Sie besitzt - selbst bei einem Felskern zwischen den Hängegletschern mit zurückwitternder Wand - eine Höhe von über $200 \mathrm{~m}$ (EUgSTER et al. 1982). Damit erreicht sie Höhen, wie sie sonst nur von der AlpenS-Seite, von der Stirn des Dora Baltea-Gletschers bei Ivrea, bekannt sind. Dort sind solche Höhen durch Überlagerung von Moränenschutt aus mehreren Kaltzeiten zu Stande gekommen. WAGNER (2001) führt diese auf Mittelmoränen zurück.

\section{Mittelmoränen-Ansätze im Bereich des Reuss-Gletschers}

\section{1 Urner Gletscher}

SE von Schattdorf (LK 1192 Schächental) hat BrÜCKNER (in BrÜCKNER et al. 1987) unter dem Schwarz Grat-Bälmeten-Grat, auf Gampelen um $1470 \mathrm{~m}$, eine Stauterrasse von durch Eis verschlepptem Bergsturzgut gezeichnet (Tab. 1). Wo das Einzugsgebiet zwischen Bälmeten und Hoch Fulen rückwärtig markanter ansteigt, fällt die Schuttterrasse aus; sie ist daher als MittelmoränenStauterrasse zwischen Schwarz Grat- und Reuss-Eis zu deuten. Die Kristallin-Blöcke von Witerschwanden (BRÜCKNER 1938, in BRÜCKNER et al. 1987) belegen das ins Schächental eingedrungene ReussEis. Der Schächen-Gletscher wurde bis Witerschwanden zurückgestaut und auf die rechte Talflanke gedrängt, was auch Erratiker bezeugen. In den Hochglazialen hatten sich auf der N-Seite des Schächentales unter den Felsgraten zwischen Guggi- und Gang- und zwischen diesem und dem Holder-Gletscher Mittelmoränen-Ansätze, als „Eggen“, gebildet (Tab. 1).

Das früh- und spätglaziale Zusammentreffen von Holder- und Rieder- Gletscher führte oberhalb Bürglen, bei Brügg (Koordinaten m NN 694,7/192,5/640), 


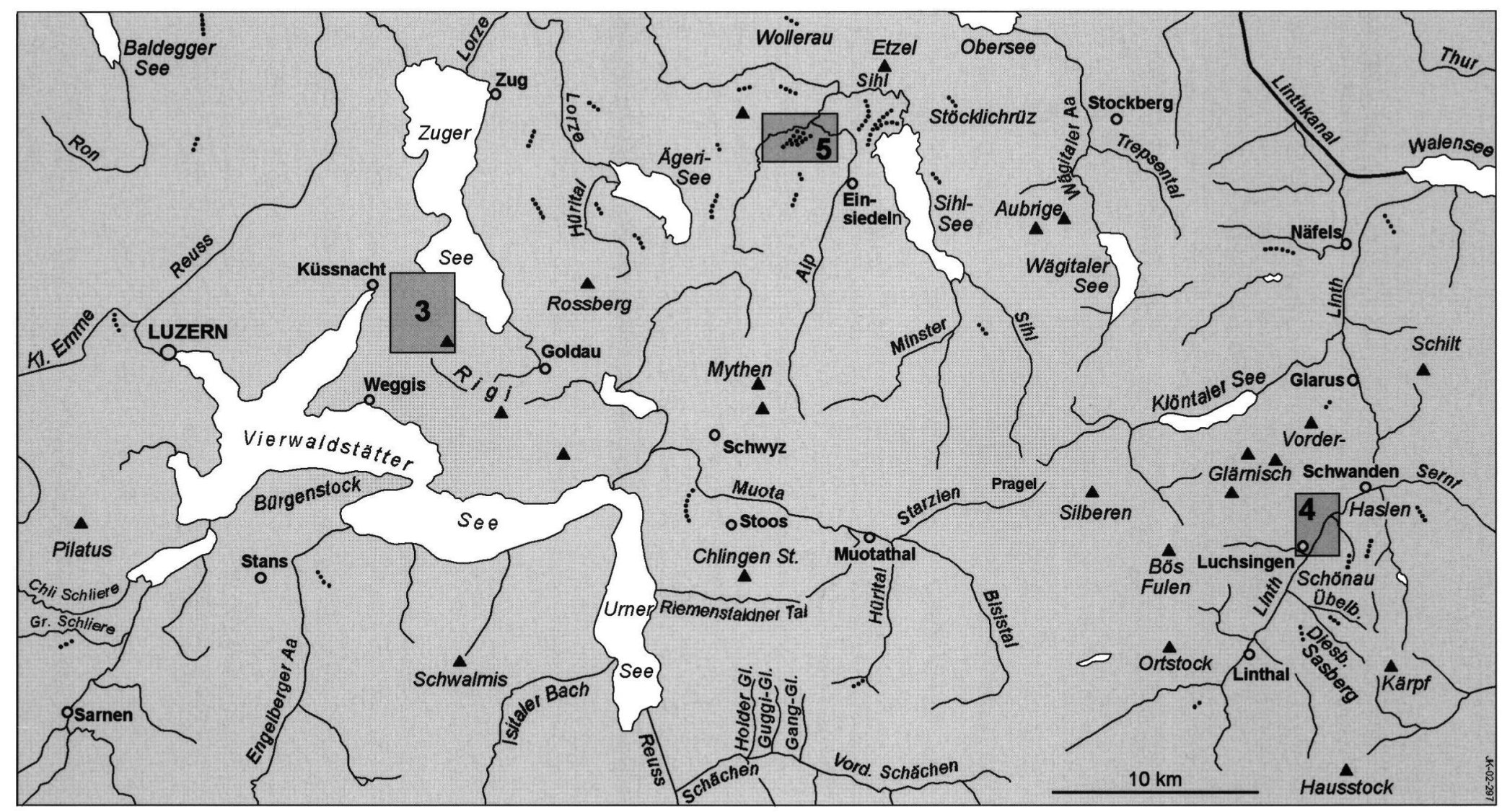

Abb. 1: Übersichtsskizze über die im Text erwähnten Mittelmoränenbereiche und die Abbildungen von Mittelmoränen in der Zentralschweiz in den Glarner Alpen. Fig. 1: Synoptic sketch of the mentioned medial moraine areas and the figures of medial moraines in Central Switzerland and in the Glarnonese Alps. 
Tab. 1: Mittelmoränen-Ansätze in der Ost- und Zentralschweiz.

Tab. 1: Beginnings of medial moraines in the Eastern and Central Switzerland.

\begin{tabular}{|c|c|c|c|c|c|}
\hline \multirow[t]{2}{*}{ Landesk. 1:25000 } & \multirow[t]{2}{*}{ Koo.-Feld/m } & \multirow[t]{2}{*}{ Ortsname } & \multicolumn{2}{|c|}{ Parental-Gletscher } & Bemerkungen, Literatur-Hinweise \\
\hline & & & links & rechts & \\
\hline 1170 Alpnach & $670 / 205 / 590$ & Bürgenstock-N & Aare-Gletscher & Reuss-Gletscher & alle Kaltzeiten, BUXTORF et al. 1916 \\
\hline 1150 Luzern & $670 / 206 / 434$ & Kehrsiten & Aare-Gl. & Reuss-Gl. & $\begin{array}{l}\text { alle Kaltzeiten, BUXTORF 1910, } \\
\text { BuXTORF et al. } 1916\end{array}$ \\
\hline 1115 Säntis & $743 / 236 / 1560$ & Chammhalden & Urnäsch-Gl. & Wissbach-Gl. & alle Kaltzeiten, HANTKE 1978, 1980 \\
\hline 1192 Schächental & $694 / 188 / 1485$ & Gampelen & Reuss-Gl. & Bärfallen-Gl. & Würm-K., BRÜCKNER et al. 1987 \\
\hline 1192 Schächental & $696 / 196 / 1980$ & Eggen $E$ & Gang-Gl. & Guggi-Gl. & alle Kaltzeiten \\
\hline 1192 Schächental & $693 / 196 / 2010$ & Eggen W & Guggi-Gl. & Holderbach-Gl. & alle Kaltzeiten \\
\hline 1192 Schächental & $694 / 196 / 1820$ & Chalberweid & Holder-Gl. & Gruon-Gl. & alle Kaltzeiten \\
\hline 1172 Muotatal & $691 / 196 / 1241$ & Eggeli & Reuss-Gl. & Gruon-G1. & Würm-Kaltzeit \\
\hline 1172 Muotatal & $691 / 199 / 1300$ & Schnäggenrüti & Buggi-Gl. & Riemenstaldner Gl. & Würm-Kaltzeit \\
\hline 1172 Muotatal & $693 / 203 / 1410$ & Holiberig & Fron-Gl. & Näppen-GI. & Würm-Kaltzeit \\
\hline 1172 Muotatal & $694 / 203 / 1210$ & Blüemlisegg & Näppen-Gl. & Muota-Gl. & Würm-Kaltzeit \\
\hline 1172 Muotatal & $707 / 195 / 160$ & Eggen & Ruosalp-Gl. & Gwalpeten-Gl. & Würm-Kaltzeit \\
\hline 1172 Muotatal & $707 / 196 / 1420$ & Waldieggen & Alpler-Gl. & Ruosalp-Gl. & Würm-Kaltzeiz \\
\hline 1173 Linthal & $707 / 195 / 1660$ & Vord. Nissegg & Gwalpeten-Gl. & Glattalp-Gl. & Würm-Kaltzeit \\
\hline 1151 Rigi & $677 / 212 / 1090$ & Seeboden & Reuss-Gl. & Rigiwald-Gl. & Würm-Kaltzeit \\
\hline 1151 Rigi & $684 / 215 / 1065$ & Rufiberg & Reuss-GI. & Gnipen-Gl. & Würm-Kaltzeit \\
\hline 1151 Rigi & $685 / 216 / 1260$ & Hagegg & Reuss-Gl. & Alpli-Gl. & Riss-Kaltzeit \\
\hline 1151 Rigi & $688 / 217 / 1230$ & Ramenegg & Zuger Arm & Ägeri-Arm d. Reuss-Gl. & Riss-Kaltzeit \\
\hline $1131 \mathrm{Zug}$ & $684 / 218 / 1080$ & Brünnli & Zuger Arm & Ägeri-Arm d. Reuss-Gl. & Riss-Kaltzeit, OTtIGER et al. 1990 \\
\hline 1171 Beckenried & $685 / 204 / 349$ & Chindli-Moräne & $\begin{array}{l}\text { Engelberger Gl. } \\
\text { Kollisionsmoräne }\end{array}$ & Reuss-Gl. & alle Kaltzeiten, HANTKE 2003b \\
\hline 1152 Ibergeregg & $691 / 213 / 1190$ & Mostelberg & Muota/Reuss-Gl. & Mostel-Gl. & Würm-Kaltzeit \\
\hline 1132 Einsiedeln & $692 / 218 / 1075$ & Chli Morgarten & Rothenth.-Arm & Ägeri-Arm d. Reuss-Gl. & Riss-Kaltzeit \\
\hline 1132 Einsiedeln & $692 / 219 / 1015$ & Tännilichrüz & Rothenth.-Arm & Ägeri-Arm d. Reuss-Gl. & Würm-Kaltzeit \\
\hline $1131 \mathrm{Zug}$ & $683 / 220 / 1018$ & Zugerberg-Grat & Zuger Arm & Ägeri-Arm d. Reuss-Gl. & Riss-K., OTTIGER et al. 1990 \\
\hline $1131 \mathrm{Zug}$ & $683 / 222 / 989$ & Hochwacht & Zuger Arm & Ägeri-Arm d. Reuss-Gl. & Riss-K., OTtiger et al. 1990 \\
\hline 1170 Alpnach & $661 / 197 / 900$ & Rütiberg & Gr. Schliere-Gl. & Brünig-Aare-Gl. & Würm-K., Buxxtorf et al. 1916 \\
\hline 1170 Alpnach & $661 / 199 / 850$ & Bodenmattli & Meisi-Gl. & Chli Schliere-Gl. & Würm-K., BuXiorr et al. 1916 \\
\hline 1150 Luzern & $663 / 205 / 1083$ & Schwandegg & Pilatus-Eis & Reuss-Gl. & Würm-K., Kopp et al. 1955, 1962 \\
\hline 1150 Luzern & $659 / 207 / 1173$ & Würzenegg & Eigen-Gl. & Aare/Reuss-Gl. & Riss-K., Kopp et al. 1955, 1962 \\
\hline 1173 Linthal & $721 / 198 / 1820$ & Hälsli & Linth-Gl. & Diesbach-Gl. & Würm-Kaltzeit \\
\hline 1173 Linthal & $721 / 199 / 1540$ & Orneli & Linth-Gl. & Diesbach-Gl. & Würm-K. $\left\{\begin{array}{l}\text { OBERHOLZER etal.1942, } \\
\text { SCHIELLY 1981 }\end{array}\right.$ \\
\hline 1173 Linthal & $723 / 199 / 1660$ & P.1666.5 & Diesbach-Gl. & Übelbach-Gl. & Würm-Kaltzeit \\
\hline 1173 Linthal & $723 / 203 / 1620$ & Mittetaghorn & Linth-Gl. & Hasler Gl. & Würm-Kaltzeit \\
\hline 1173 Linthal & $721 / 203 / 610$ & Luchsingen & Bösbächi-Gl. & Linth-Gl. & Würm-Kaltzeit \\
\hline 1173 Linthal & $723 / 203 / 560$ & Nidfurn & Guppen-Gl. & Linth-Gl. & Würm-Kaltzeit \\
\hline 1173/1174 Elm & $725 / 204 / 1260$ & Sedel & Linth-Gl. & Änetseeben-Gl. & Würm-Kaltzeit \\
\hline $1174 \mathrm{Elm}$ & $726 / 205 / 1660$ & Hellhom & Nideri-Gl. & Sernf-Gl. & Würm-Kaltzeit \\
\hline 1153 Klöntal & $722 / 210 / 1000$ & Stöckli & Bruch-Gl. & Linth-Gl. & präwürmz. Kaltzeit? \\
\hline 1153 Klöntal & $712 / 208 / 1120$ & Schwammhöchi & Richisauer G1. & Rossmatter Gl. & Würm-Kaltzeit \\
\hline 1153 Klöntal & $710 / 208 / 1235$ & Gampeleggen & Schwialp-Gl. & Chlön-Gl. & Würm-Kaltzeit \\
\hline 1153 Klöntal & $719 / 217 / 1350$ & Boggenberg & Schwändi-Gl. & Obersee-Gl. & alle Kaltzeiten \\
\hline 1133 Walensee & $725 / 219 / 640$ & Vor dem Wald & Linth-Gl. & Walensee-A. d. Rh.-GI. & alle K., HerB \& Dollfus 2003 \\
\hline 1153 Klöntal & $714 / 217 / 1490$ & Schwarzenegg & Wägitaler Gl. & Trepsen-(Chälen)-Gl. & alle Kaltzeiten \\
\hline 1132 Einsiedeln & $704 / 223 / 1248$ & Wissegg & Chnüweg-Gl. & Linth/Rhein-Gl. & Riss-Kaltzeit \\
\hline 1132 Einsiedeln & $703 / 224 / 1150$ & Beristofel & Sihl-Gl. & Linth/Rhein-Gl. & Riss-Kaltzeit \\
\hline 1132 Einsiedeln & $700 / 223 / 939$ & Ober Waldweg & $\begin{array}{l}\text { Sihl-Gl. } \\
\text { Kollisionsmoräne }\end{array}$ & Linth/Rhein-Gl. & Würm- + ältere Kaltzeiten \\
\hline 1132 Einsiedeln & $701 / 218 / 1000$ & Breiten & Gross-Gl. & Sihl-Gl. & Würm- + ältere Kaltzeiten \\
\hline 1132 Einsiedeln & $703 / 220 / 970$ & Fuchsberg & Sihl-Gl. & Miesegg-Gl. & Würm- + ältere Kaltzeiten \\
\hline 1152 lbergeregg & $700 / 209 / 1210$ & Änglisfang & Fallen-Gl. & Minster-Gl. & Würm-Kaltzeit \\
\hline 1152 Ibergeregg & $701 / 209 / 1300$ & Bueffen & Minster-Gl. & Chäswald-Gl. & Würm-Kaltzeit \\
\hline 1152 lbergeregg & $695 / 212 / 1350$ & Brüschrain & Nätschberg-Gl. & Grossbrechenstock-Gl. & alle Kaltzeiten \\
\hline
\end{tabular}




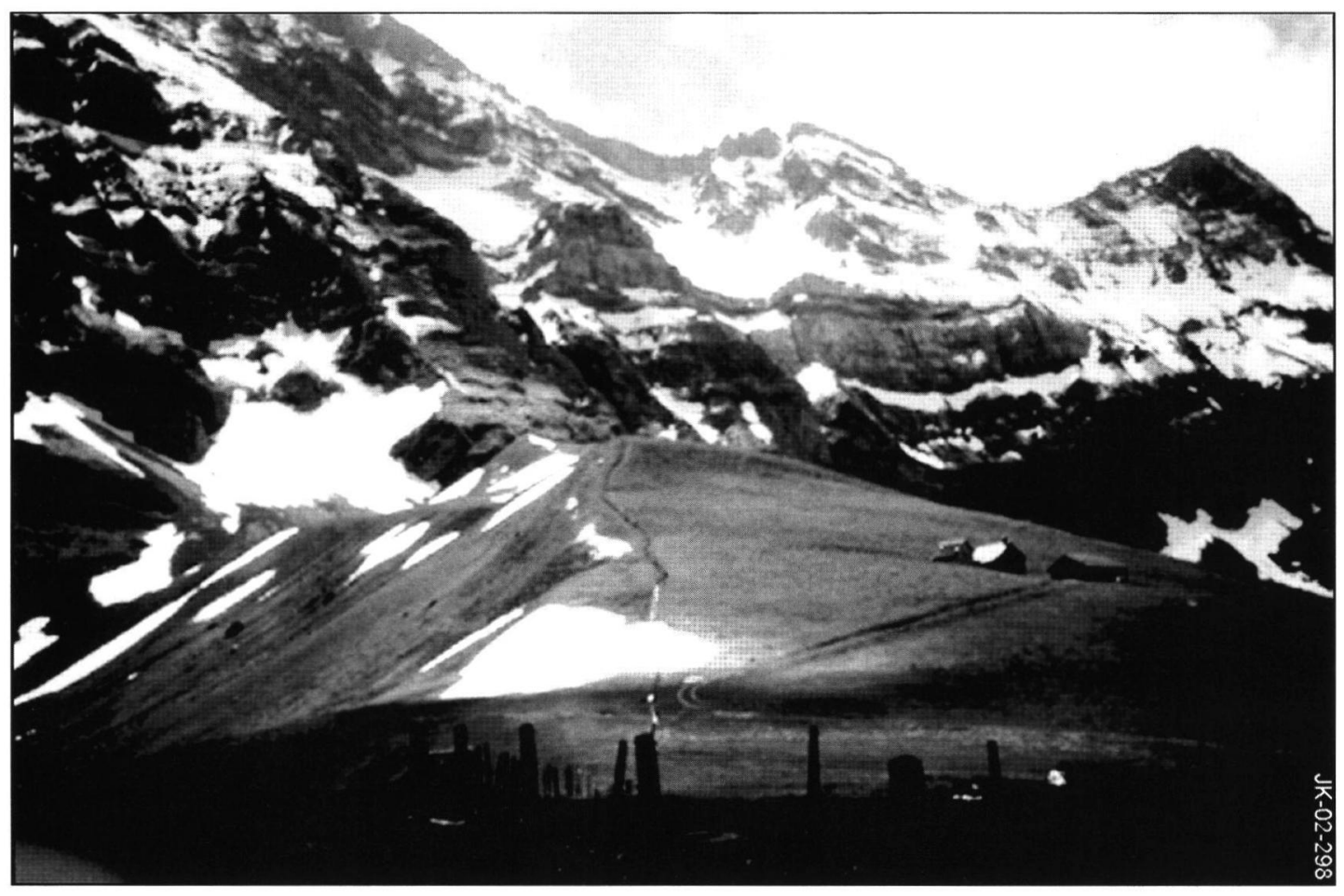

Abb. 2: Die Mittelmoräne der Chammhalden von der Chammhaldenhütte aus mit Girenspitz und Grenzchopf in der N-Kette des Säntisgebirges (Frontalansicht).

Fig. 2: The medial moraine of the Chammhalden from the Chammhalden cabin with Girenspitz and Grenzchopf in the Northern chain of the Säntis mountains in the background (Frontal view).

zum Stau der Schotter-Terrassen von Breitäbnet und Ämmerten; sie bekunden die Lage des im frühund spätglazialen Chindli/Ibacher- bzw. Ingenbohler Stand (HANTKE 2003b) ins untere Schächental eingedrungenen Reuss-Gletschers (Tab. 2b).

\subsection{Muota-Gletscher}

Zwischen Reuss- und Muota-Gletscher haben sich E von Brunnen, im westlichen Stooswald oberhalb der Luftseilbahn-Talstation Morschach-Stoos, Mittelmoränen abgelagert. Dies wird durch das plötzliche Ausfallen kristalliner Reuss-Erratiker gegen das Muotatal belegt (LK 1172 Muotatal).

$\mathrm{S}$ des Stoos hat sich auf dem Holiberig eine Mittelmoräne zwischen Fronalp-und Näppen-Gletscher gebildet. Der Blüemlisegg-Wall (694/203/1204) E des Stoos ist als Mittelmoräne zwischen diesem und dem Muota-Gletscher zu deuten (Tab. 1).
Im hintersten Muotatal wurden zwischen Alpler Stock und dem Ruosalper Tal, zwischen diesem und Gwalpeten sowie am NW-Fuss des Märenspitz, im steilen Schuttgrat von Vorderist Nissegg (708/195), Mittelmoränengut zwischen dem von Gwalpeten und Glattalp abgestiegenen Eis geschüttet.

\subsection{Vierwaldstätter See-Reuss- und ver- einigter Muota/Reuss-Gletscher}

Mittelmoränen-Wälle haben sich an der NW-Flanke der Rigi, in der Seeboden-Moräne (Abb. 1 und 3) und $\mathrm{N}$ des Rossberg, auf dem Rufiberg erhalten. Da an beiden Orten frühe spätwürmzeitliche Moränen weit herab, auf der N-Seite der Rigi bis gegen den Zuger See reichen, können sie kaum Seitenmoränen eines Muota/Reuss-Gletschers sein, wie bisher angenommen wurde: sie bildeten sich zwischen Eis von der Rigi bzw. vom Rossberg und dem Reuss- 


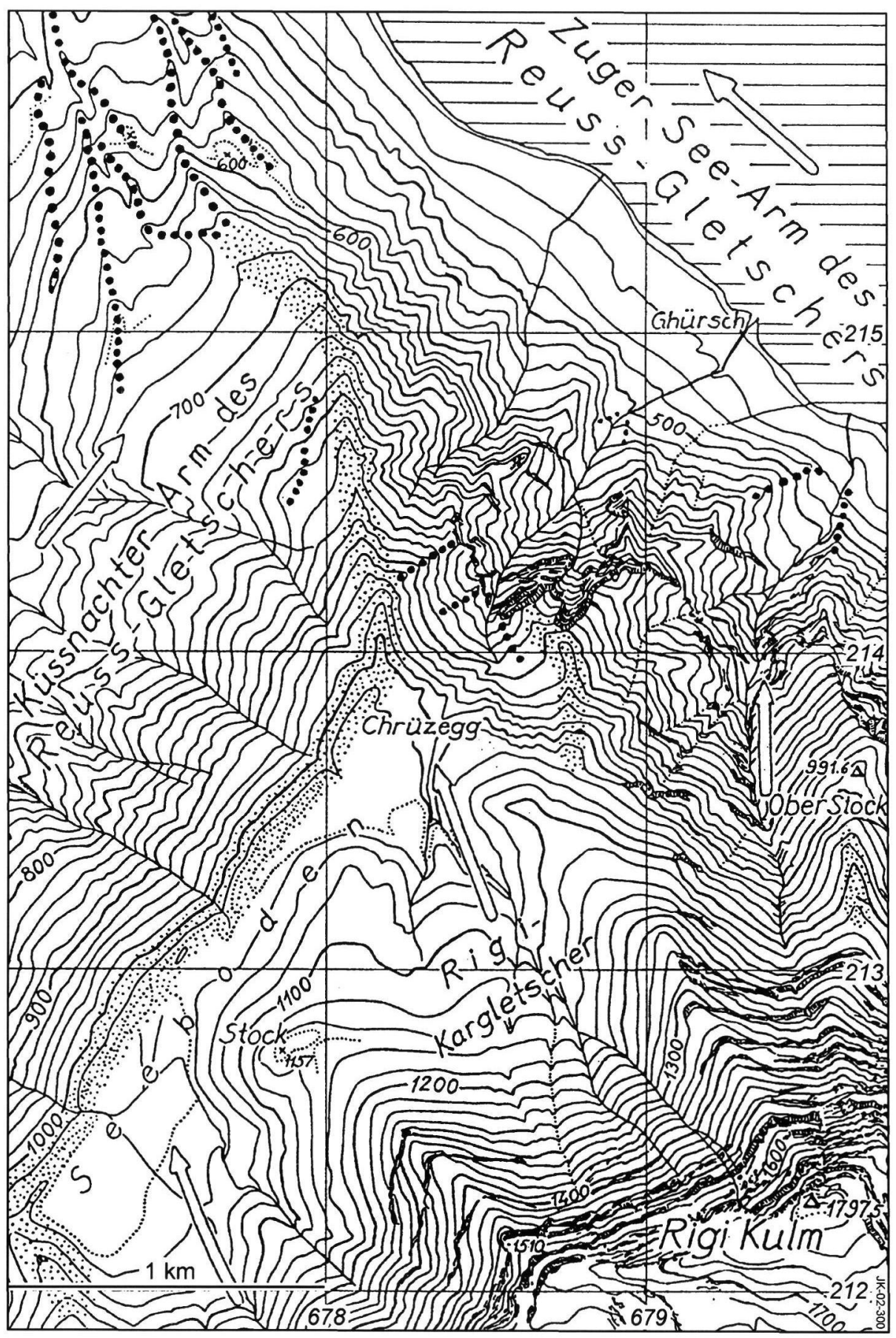

Abb. 3: Die Seeboden-Mittelmoräne (punktiert) zwischen dem Küssnachter Arm des Reuss-Gletschers und dem RigiLokaleis; dicke Punktzeilen: die frühe Spätwürm-Moräne des Rigi-Eises gegen den Zuger See. LK 1151 Rigi.

Fig. 3: The medial moraine of Seeboden (dotted) between the Küssnacht branch of the Reuss glacier and local ice from the Rigi mountain. The early Late-Würmian moraine of the Rigi ice against the Lake Zug is shown by a heavy-dotted line. 
bzw. Muota/Reuss-Gletscher. Dabei hat das in den Höchstständen mächtigere Taleis das Zufliessen von Lokaleis verhindert (Tab. 1).

Auf der E-Seite des Zuger Sees, auf dem Walchwiler Berg, zeichnet sich zwischen zwei flachen spätrisszeitlichen Moränenwällen eine ebenso flache risszeitliche Mittelmoräne auf Brünnli-Nollen (Atlasblatt 1131 Zug) ab. Die Schotter an der NE-Seite des Grossmattstollen ( $\mathrm{N}$ des Rossberg, Tab. 1) sind als verschwemmte prärisszeitliche Mittelmoräne von Muota-Reuss-Gletscher-Armen zu deuten.

Der Wall von Mostel ist als Mittelmoräne zwischen dem Rothenthurmer Arm des Muota/Reuss-Gletschers und von Hochstuckli und Engelstock zugeflossenem Eis zu interpretieren (Tab. 1).

Zwischen Chli Morgarten und der Molasserippe von St. Jost haben sich zwischen Ägeri- und Rothenthurmer Arm des gegen $\mathrm{N}$ abgedrängten Muota/Reuss-Gletschers in unterschiedlichen Höhenlagen verschieden alte Mittelmoränen gebildet. Die verkitteten und randlich verstürzten Schotter des Ratengütsch (1119.2 m) mit Verrucano-Erratikern, auf Grund der Höhenlage subalpine Deckenschotter, liegen zwischen Rothenthurmer und Ägeri-Arm des Muota/Reuss-Gletschers und dem zur Zeit der grössten Vergletscherungen von $\mathrm{N}$ eingedrungenen Linth/ Rhein-Gletscher (LK 1132 Einsiedeln). Weitere ältere Mittelmoränen zwischen Rothenthurmer Arm des Muota/Reuss-Gletschers und Alp-Gletscher haben sich auf Chrüzweid-Hundwileren-Chatzenstrick gebildet (Tab. 1).

\section{Mittelmoränen-Ansätze des Linth-Gletschers}

E von Linthal (LK 1173 Linthal) setzt eine höchste Mittelmoräne auf Hälsli (1772 m) N der Alp Sasberg zwischen Linth- und Diesbach-Gletscher um $1840 \mathrm{~m}$ ein, eine tiefere beginnt bei Orneli um $1600 \mathrm{~m}$ (Tab. 1).

Den Grat zwischen Diesbach- und Übelbachtal (LK 1173 Linthal) haben OBERHOLzer (in OBERHOLZER \& Heim, Alb 1910, Oberholzer et al. 1942) und Schielly (1981) verschieden, aber beide als anstehend kartiert. Die Neukartierung hat jedoch gezeigt, dass der distale Grat von P. 1765 bis $1540 \mathrm{~m}$ aus Moränengut besteht; er stellt somit eine Mittel- moräne zwischen Diesbach- und ÜbelbachGletscher dar (Tab. 1).

Zwischen Linth-Gletscher und Bächi-Gletscher löst sich auf $1170 \mathrm{~m}$ eine Mittelmoräne, die sich gegen das Luchsinger Tobel bis über Schlatt verfolgen lässt. $\mathrm{N}$ von Luchsingen verlaufen Wallreste am linken Hangfuss gegen Leuggelbach. Weiter NE vermochte der Guppen-Gletscher den Bächi/Linth-Gletscher noch $\mathrm{zu}$ erreichen und wurde von diesem ebenfalls nach NW abgedrängt. Die vorgelagerte, durch Bergsturzgut gestaute und von Schmelzwässern zerschnittene Schotterflur von Leuggelbach-Nidfurn-Thon bekundet verschwemmtes spätglaziales und wohl noch älteres Mittelmoränengut (Abb. 1 und 4).

Im hinteren Klöntal ist die bisher als Seitenmoräne des Rossmatter Gletschers gedeutete Moräne, die den Talboden der Richisau abdämmt, in ihrem distalen Teil als Mittelmoräne zu interpretieren. Zweifelsfrei sind im Tal der Richisauer Chlön sich einstellende Mittelmoränen, jene des Gampeleggen, zwischen nördlichem Silberen-Eis und einer Zunge, die zwischen Fläschenspitz und Brüschalp von NW zugeflossen ist, sowie der trennende Wall auf Mittler Schwialp (Tab. 1).

Im vordersten Glarnerland (LK 1153 Klöntal) lösen sich am Bärensolspitz um 1360 m Mittelmoränen zwischen Obersee- und Schwändi-Gletscher; gegen E fallen sie über den Boggenberg auf $1240 \mathrm{~m}$ ab und bestätigen die zwischen Walensee-Arm des Rhein- und Linth-Gletscher festgestellten Eishöhen (Tab. 1).

\section{Mittelmoränen-Ansätze des Linth/Rhein- Gletschers und seiner Zuflüsse}

Über dem W-Ende des Walensees treten an der Konfluenz von Linth- und Walensee-Arm des Rhein-Gletschers weitere Mittelmoränen-Ansätze auf: ein von Erratikern begleiteter Ansatz setzt bei Rütisbrunnen um $950 \mathrm{~m}$ ein und steigt über Vor dem Wald (LK 1134 Walensee) gegen das Escher Kanal-Knie ab (Ochsner 1969, Tab. 1).

Im Trepsental, einem Seitenast des Wägitales, setzt auf Schwarzenegg (LK 1153) um $1480 \mathrm{~m}$ vor der Chälen, dem Kar zwischen Schiberg und Bockmattli, eine persistente Mittelmoräne zwischen Wägitalerund Trepsen-Gletscher an. Dieser erhielt noch im 


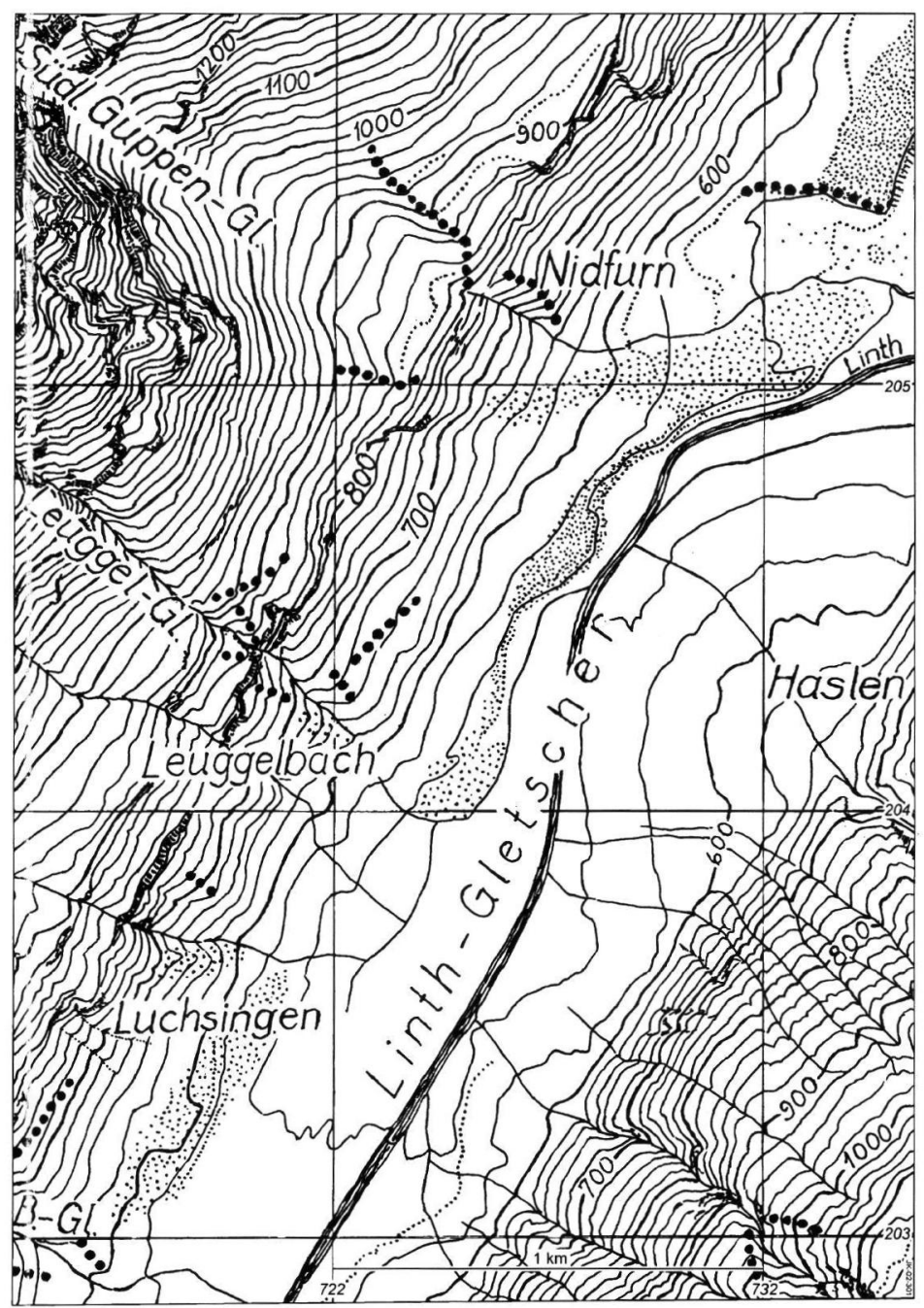

Abb. 4: Die im Linthtal auftretende Mittelmoräne (punktiert) zwischen Linth- und Bächi-Gletscher und zwischen diesem und dem südichen Guppen-Gletscher; feinpunktiert: Schotter, verschwemmte Mittelmoräne; dicke Punktzeile: Jüngere Wieder rorstösse. LK 1173 Linthal.

Fig. 4: The medial moraine (dotted) in the Linth valley between the Linth- and Bächi glaciers and between the latter and the southern Guppen glaciers; fine dotted: gravel, floated medial moraine. Younger readvances are shown by heavy dots.

Spätglazial einen Zuschuss vom Bockmattli und von weiter E zufliessenden Gletschern (Tab. 1).

Präwürmzeitliche Mittelmoränen wurden am Voralpenrand auf Stöcklichrüz und auf Wissegg zwischen austretenden Kargletschern und dem Linth/Rhein-Gletscher und weiter W, auf dem Grat P. 1202-Beristofel-Eggli, zwischen Linth- und SihlGletscher gebildet (LK 1132 Einsiedeln).
Würmzeitliche Mittelmoränen haben sich zwischen dem über die Sihl vorgedrungenen Linth-Gletscher und von der Höhronen-Kette zugeflossenen Eiszungen gebildet. Der zwischen Etzel und Höhronen von $\mathrm{N}$ ins obere Sihltal eingedrungene Linth/ Rhein-Gletscher staute den Sihl-Gletscher am NWEnde des Sihlsees zu mächtigen, gegen einander gestoßenen Stirnmoränen, eine Kollisionsmoräne. Diese ist kaum nur das Werk eines würmzeitlichen 
Eisvorstosses zum Maximalstand, an ihrem Aufbau waren schon weit ältere Stände beteiligt. Nach Überwindung dieser Randlage vermochte Linth/ Rhein-Eis in den grössten Kaltzeiten kurzfristig tiefer ins Sihltal einzudringen, was einzelne Verrucano-Blöcke belegen.

Neben der gestauten Stirnmoräne des Sihl-Gletschers haben sich zwischen Gross- und SihlGletscher sowie zwischen diesem und dem bei Willerzell mündenden Miesegg-Gletscher von Büelhöchi (1425.1 m) und Rinderweidhorn (1316.8 m) mächtige Stauschuttmassen und Mittelmoränen gebildet (LK 1132 Einsiedeln, Tab. 1).

Im Minstertal hat $\mathrm{N}$ des Bueffengaden eine Mittelmoräne eingesetzt. Sie setzt sich ein Stück weit als linke Seitenmoräne des von der Mördergruebi zugeflossenen Parentalgletschers ins Chäswaldtobel fort. Im hinteren Alptal verläuft unter der Brüschrainhöchi von P. 1362 ein breiter, fast horizontal verlaufender Schuttkörper zum Brüschrain. Dieser wurde über alle Kaltzeiten sukzessive höher geschüttet; er ist als persistente Mittelmoräne zwischen den beiden Gletschern vom Nätschboden-Brüschrainhöchi und BrüschrainhöchiGrossbrechenstock zu deuten (Tab. 1).

\section{Mittelmoränen-Endaufschüttungen}

Die bisher vorgelegten Beispiele haben Strukturen an den Ansätzen von Mittelmoränen gezeigt. Diese bestehen aus Seitenmoränengut der beiden Parentalgletscher, das den Anschluss an die Mittelmoräne verpasst hat und an ihrem Ursprung auf festem Boden liegen blieb.
Die weiter zu betrachtenden Strukturen bestehen aus Moränengut, das von diesen Stellen auf dem Eis als Schuttförderband, als Mittelmoräne, verfrachtet worden ist. Mittelmoränen können ihre Individualität über weite Fliessstrecken beibehalten, was bei heutigen alpinen Gletschersystemen zu beobachten ist. Irgendwo, seitlich an einem Nunatak, spätestens am Zungenende, erreichen sie den Eisrand und schütten dort charakteristische Ablagerungen, wie sie WAGNer (1997) typisiert hat. Dabei kann das Mittelmoränengut ganz oder teilweise durch Schmelzwässer und Starkniederschläge verschwemmt werden und zu eisrandnahen Schottern führen. Diese können je nach dem Eisrand in irgend einer Höhe liegen. Damit kann die Höhenlage von Moränen und Schottern - entgegen der bisherigen Auffassung - kein Kriterium für ihr Alter sein.

\subsection{Mittelmoränen-Endaufschüttungen auf Molasse-Hochflächen (Tab. 2a)}

Hochgelegene Endaufschüttungen von Mittelmoränen über verschwemmtem Mittelmoränengut setzen W des Höhronen auf SchurtannenweidBrämerhöchi-Kloster Gubel zwischen Linth/ Rhein- und Reuss-Eis ein (LK 1131 Zug). Je weiter die alpinen Gletscher ins Schweizer Mittelland vorstießen, umso stärker schuttbeladen traten sie in den Ablationsbereich. Dabei sind sie vielfach auf primären Molasse-Hochflächen auf Grund gelaufen. Vom Kloster Gubel ist bei hohen Eisständen zu verschiedenen Kaltzeiten eine Mittelmoräne zwischen Reuss- und Linth-Gletscher über die Baarburg (LK 1131), eine Kuppe von Oberer Süßwassermolasse mit aufgesetztem verkitteten Schot-

Tab. 2a: Mittelmoränen-Endaufschüttungen auf Molasse-Hochflächen im Schweizer Mittelland.

Tab. 2a: On Molasse Highplateaus agrounded medial moraines in the Swiss Lowland.

\begin{tabular}{|llllll}
\hline Landesk. 1:25000 & Koo.-Feld/m & Ortsname & \multicolumn{2}{c|}{ Parental-Gletscher } & Bemerkungen, Literatur-Hinweise \\
& & & links & \multicolumn{1}{l}{ rechts } \\
\hline 1132 Einsiedeln & $696 / 228 / 550$ & Studenbüel & Linth-Parental-Gl. & Linth/Rhein-Gl. & Pseudodrumlin \\
1132 Einsiedeln & $698 / 229 / 410$ & Bächau & Linth-Parental-Gl. & Linth/Rhein-Gl. & wohl präwürmz. K., AEPPLI 1894 \\
1112 Stäfa & $691 / 233 / 450$ & Halbinsel Au & Sihl-Gl. & Linth/Rhein-Gl. & verkittet, präwürmzeitl. K., AEPPLI 1894 \\
1132 Einsiedeln & $694 / 229 / 570$ & Alt Wädenswil & Sihl-Gl. & Linth/Rhein-Gl. & ältere K., HANTKE et al. 2000c \\
1131 Zug & $684 / 228 / 683$ & Baarburg & Muota/Reuss-Gl. & Linth/Rhein-Gl. & Riss-K., OTTIGER et al. 1990 \\
1111 Albis & $683 / 233 / 910$ & Albishorn & Muota/Reuss-Gl. & Linth/Rhein-Gl. & ältere K. (als Höherer Deckenschotter) \\
1110 Hitzkirch & $666 / 233 / 865$ & Lindenberg & Reuss-Gl. & Reuss-Gl. & ältere K., MUHLBERG 1910 \\
\hline
\end{tabular}


ter, zur Albis-Kette verlaufen. Natur und Alter des Baarburg-Schotters wurden über ein Jahrhundert diskutiert (Aeprli 1894; Frei 1912; Heim 1894 , 1913, 1919; HantKe 1961). Die Schotterkappe bekundet alten, verschwemmten MittelmoränenSchutt. Ebenso deuten die wallgekrönten verkitteten Schotter unter der Ruine Alt Wädenswil (LK 1132 Einsiedeln) auf präwürmzeitliches, verschwemmtes Mittelmoränengut. Die Deutung als verschwemmtes Mittelmoränengut ermöglicht eine neue Interpretation. Die Hypothese rückgesenkter Deckenschotter (Heim 1894, Aeppli 1894), sukzessive erosive Reliefeintiefung oder junge tektonische Verstellungen werden hinfällig.

Als Mittelmoräne zwischen Wägitaler- und Linth/ Rhein-Gletscher ist der drumlinartige Obermoränenhügel des Studenbüel NW von Wollerau zu deuten (Tab. 2a). Ebenso deuten die Moränen- bzw. Schotter-Vorkommen der Halbinsel Bächau (LK 1132 Einsiedeln) sowie die verkitteten Schotter mit reichlich Verrucano-Gut der von Moräne mit Erratikern bedeckten Halbinsel Au (LK 1112 Stäfa) am linken Zürichsee-Ufer auf Mittelmoränen.

\subsection{Stauschutt-Terrassen und Mittel- moränen-Endaufschüttungen in der Zentralschweiz (Tab. 2b)}

Endaufschüttungen als vorgeschobene „Schuttfinger“ der einzelnen Mittelmoränenkämme nach ihrem langen Weg auf dem Eis finden sich im voralpinen Bereich in Hochlagen, so bei dem über den Pass von Rothenthurm übergeflossenen Arm des Muota/Reuss-Gletschers. Das in Abb. 5 wiedergegebene Kurvenbild entspricht genau dem von
Wagner (1997, Abb. 14) für die Enden der Mittelmoränenfinger des Aaregletschers bei Bern festgehaltenen Bild.

Im äussersten würmzeitlichen Stand reichte der Rothenthurmer Arm des Muota/Reuss-Gletschers von SW bis an die Terrasse von Bennau. Davor lagerte er auf seiner Oberfläche mitgeführten Mittelmoränenstränge $a b$, die gegen das Zungenende fingerförmig auseinander strahlen (Abb. 1 und 5, Tab. 2b). Im entsprechenden Stand des zwischen Etzel und Höhronen eingedrungenen Linth/RheinGletschers floss Eis über den Sattel P. 911 E des Altberg ins untere Alptal, durch dieses von der NE-Seite an die Stauterrasse von Bennau und weiter bis Biberbrugg, wo es kleine Wallreste hinterliess.

Tabelle 1 fasst die bedeutendsten in den Kartengebieten festgestellten Mittelmoränenansätze.

Tabellen 2a und 2b fassen Stauterrassen und Endaufschüttungen zusammen.

Tab. 2b: Stauterrassen alpiner Randlagen und Mittelmoränen-Endaufschüttungen in der Zentralschweiz.

Tab. 2b: Barred terrasses on alpine border positions und terminal of medial moraines in the Central Switzerland.

\begin{tabular}{|llllll|}
\hline Landesk. 1:25000 & Koo.-Feld/m & Ortsname & \multicolumn{2}{c|}{$\begin{array}{c}\text { Parental-Gletscher } \\
\text { rechts }\end{array}$} & Bemerkungen, Literatur-Hinweise \\
\hline 1192 Schächental & $694 / 192 / 740$ & Ämmerten & Reuss-Gletscher & Schächen-Gl. & Würm-Stauterrasse, BRÜCKNER et al.1987 \\
1192 Schächental & $695 / 192 / 780$ & Breitäbnet & Schächen-G & Holderbach-Gl. & W.-Stauterrasse, BRÜCKNER et al.1987 \\
1192 Schächental & $694 / 192 / 740$ & Ried & Holderbach-Gl. & Reuss-Gl. & W.-Stauterrasse, BRÜCKNER et al.1987 \\
1132 Einsiedeln & $696 / 222 / 850$ & Moos & Rothenth. Arm des & Muota/Reuss-Gl. & W.- Zungen-Ende, HANTKE et al. 2001c, \\
& & & & & Abb. 5 \\
\hline
\end{tabular}




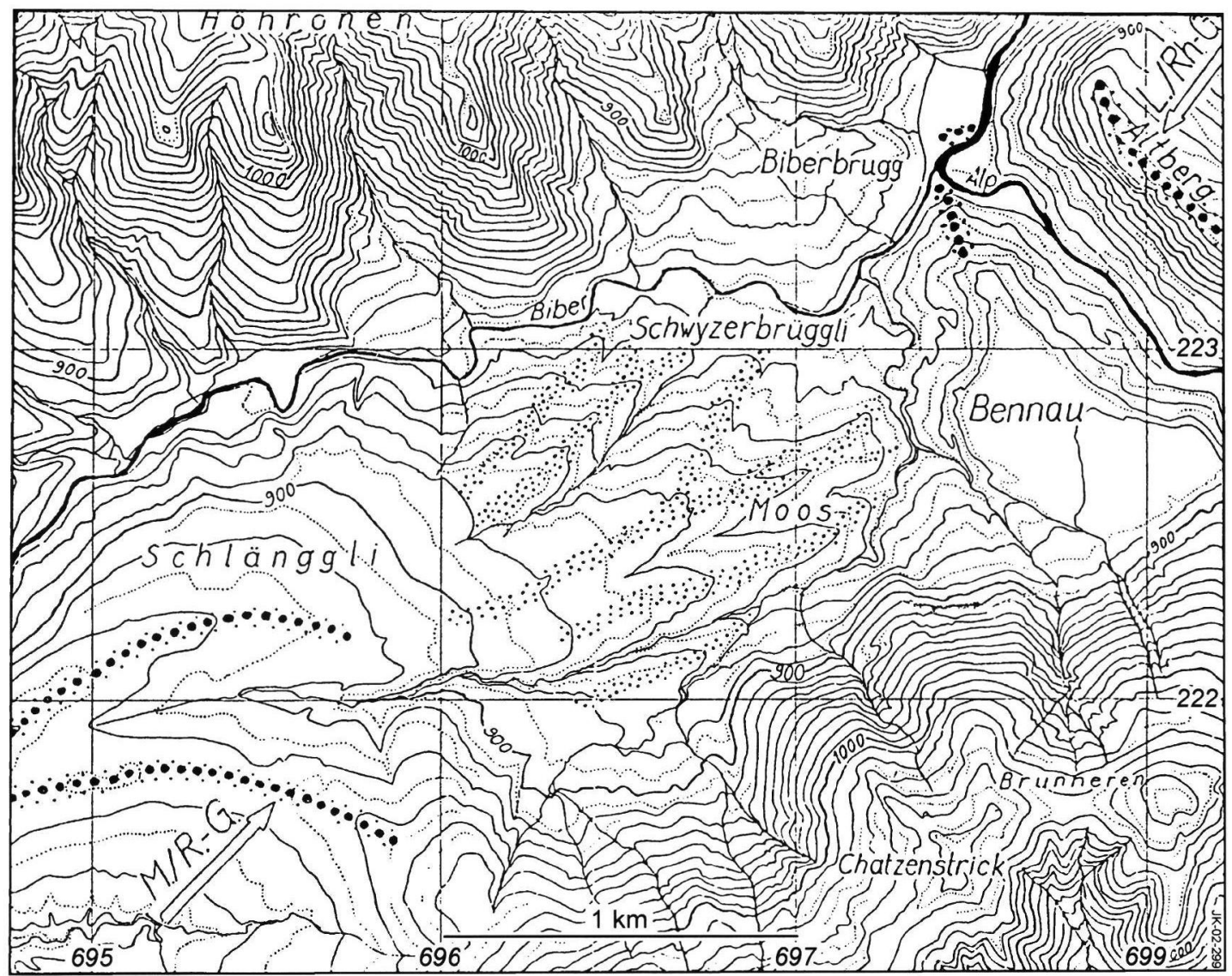

Abb. 5: Die Mittelmoränen-Endaufschüttungen des Rothenthurmer Armes des Muota/Reuss-Gletschers (punktiert) mit ihren Schuttfingern während des würmzeitlichen Maximalstandes; jüngerer Maximalstand mit dicken Punkten. LK 1132 Einsiedeln.

Fig. 5: The accumulations of terminal medial moraine of the Rothenthurm branch of the Muota/Reuss glacier: dotted; the younger maximum stages: haevy dotted.

\section{Literatur und Karten}

AEprLi, A. (1894): Erosionsterrassen und Glazialschotter in ihrer Beziehung zur Entstehung des Zürichsees mit Geologischer Karte des Gebietes zwischen Zürichsee und Zugersee 1: 25000. - Beitr. geol. Karte Schweiz, N.F. 4.

BRƯCKNER, W. (1938): Die Quartärbildungen im oberen Schächental, Kt. Uri. - Eclogae geol. Helv., 30/2 (1937): 273-292.

- \& ZBInden, P. (1987): Blatt 1192 Schächental. Geol. Atlas Schweiz 1: 25000. - Schweiz. geol. Komm.

Buxtorf, A. (1910): Geologische Karte der Pilatus-Bürgenstock-Rigihochfluhkette, Blatt 2: Bürgenstock, 1: 25000, mit Erläuterungen. - Geol. Spez.-Karte, 27b - Schweiz. geol. Komm.
- , Tobler, A., Niethammer, G., Baumberger, E., Arbenz, P. \& Staub, W. (1916): Geologische Vierwaldstätterseekarte 1: 50000. - Geol. Spez.-Karte, 66a. - Schweiz. geol. Komm.

Eugster, H., Forrer, M., Fröhlicher, H., Kempf, Тh., Schlatter, L., Blaser, R., Funk, H., Langenegger, H., Spoerri, M. \& Habicht, K. (1982): Blatt 1115 Säntis. - Geol. Atlas Schweiz 1: 25000. - Schweiz. geol. Komm.

FreI, R. (1912): Monographie des schweizerischen Deckenschotters mit Geologischer Karte des Lorzetobel-Sihlsprung-Gebietes (Kt. Zug) 1: 25000. - Beitr. geol. Karte Schweiz, NF. 37.

Funk, H., Habicht, K., Hantke, R., Pfiffner, O. A., mit Beiträgen von KoBel, M. (2000): Erläuterungen zu Blatt 1115 Säntis. - B.-A. Wasser u. Geologie, Bern. 
HANTKE, R. (1961): Zur Quartärgeologie im Grenzbereich zwischen Muota/Reuss- und Linth/Rheinsystem. - Geogr. Helv., 16/4: 212-223.

- (1978, 1980, 1983): Eiszeitalter, 1, 2, 3. - Thun (Ott); 1992 Landsberg/Lech (ecomed).

- (2000a): Blatt 1172 Muotatal mit Ostrand von Blatt 1171 Beckenried. - Geol. Atlas Schweiz 1: 25000, mit Erläuterungen, dep. Geol. Landesaufn., Bern.

- et al. (2000b): Blatt 1151 Rigi mit Nordteil von Blatt 1171 Beckenried. - Geol. Atlas Schweiz 1: 25000, mit Erläuterungen, dep. Geol. Landesaufn., Bern; erscheint 2003 B.A. Wasser u. Geologie.

- et al. (2000c): Blatt 1132 Einsiedeln mit Anhängsel von Blatt 1112 Stäfa. - Geol. Atlas Schweiz 1: 25000, mit Erläuterungen, dep. Geol. Landesaufn., Bern.

— et al. (2000d): Blatt 1173 Linthal mit Anhängsel von Blatt 1193 Tödi. - Geol. Atlas Schweiz 1: 25000, mit Erläuterungen, dep. Geol. Landesaufn., Bern.

- et al. (2000e): Blatt 1153 Klöntal. Geol. Atlas Schweiz 1: 25000, mit Erläuterungen, in Vorber.

- et al. (2000f): Blatt 1152 Ibergeregg. - Geol. Atlas Schweiz 1: 25000, mit Erläuterungen, in Vorber. Geol. Landesaufn.; Bern.

- (2003a): Eiszeitliche Mittelmoränen in der Zentralschweiz und in den westlichen Glarner Alpen Ber. schwyz. natf. Ges., 14.

- (2003b): Unterseeische Moränen im Vierwaldstätter See. - Ber. Schwyz. Natf. Ges., 14.

- \& WaGner, G. (2003): Mittelmoränen als Schuttlieferanten der Thurgauer Deckenschotter. - Mitt. thurg. natf. Ges., im Druck.

Heim, A. (1894): Die Entstehung der alpinen Randseen. - Vjschr. natf. Ges. Zürich, 39/1: 66-84.

- (1913). Über die Rückläufigen Deckenschotter. Eclogae geol. Helv., 13/5: 682-683.
- (1919): Geologie der Schweiz, 1. - Tauchnitz, Leipzig.

Herb, R. \& Dollfus, S. (2003): Blatt 1134 Walensee. Geol. Atlas Schweiz 1: 25000. - Bodenanstalt Wasser u. Geologie, Bern.

Kopp, J., Bendel, L. \& Buxtorf, A. (1955): Blatt Luzern. Geol. Atlas Schweiz 1: 25000. - Schweiz. geol. Komm.

_ mit Beiträgen von Buxtorf, A. (1962): Erläuterungen zu Blatt Luzern. - Schweiz. geol. Komm.

MühlberG, F. (1910): Geologische Karte der Umgebung des Hallwilersees und des obern Winen- und Surtales 1:25000. - Geol. Spez.-Karte, 54 - Schweiz. geol. Komm.

Oberholzer, J. et al. (1942): Geologische Karte des Kantons Glarus 1: 50000. - Geol. Spez.-Karte, 117 Schweiz. geol. Komm. (1984 unveränd. Neudr.).

- \& Heim, A. (1910): Geologische Karte der Glarneralpen 1: 50000. - Geol. Spez.-Karte, 50 - Schweiz. geol. Komm.

OCHSNER, A. (1969, 1975): Blatt 1133 Linthebene. Geol. Atlas Schweiz 1: 25000, mit Erläuterungen. Schweiz. geol. Komm.

Ottiger, R., Freimoser, M., Jäckli, H., Kopp, J. \& Múller, E. (1990): Blatt 1131 Zug. - Geol. Atlas Schweiz 1: 25000 - Landeshydrol. u. -geol. Bern.

Schielly, H.-P. (1981): Geologische Karte des westlichen Freiberges (Kt. Glarus), 1: 10000. - Natf. Ges. Kt. Glarus.

WaGneR, G. (1986): Die eiszeitlichen Moränen von Aare- und Rhonegletscher im Gebiet des Worblentals bei Bern. - Mitt. natf. Ges. Bern, N. F. 43: 63-110.

- (1997): Eiszeitliche Mittelmoränen im Berner Mittelland. - Mitt. natf. Ges. Bern, N. F. 54: 91-137.

- (2001): Mittelmoränen eiszeitlicher Alpengletscher in der Schweiz. - Eclogae geol. Helv., 94/2: 221-235. 